\title{
Early Gastric Cancer Exfoliating into Gastric Lavage (GL1 EGC) Shows a More Aggressive Behavior and Poorer Survival Compared to the Non-Exfoliative Counterpart (GL0 EGC)
}

\author{
EDOARDO VIRGILIO ${ }^{1}$, ENRICO GIARNIERI ${ }^{2}$, MARIA ROSARIA GIOVAGNOLI ${ }^{2}$, \\ MONICA MONTAGNINI ${ }^{2}$, ANTONELLA PROIETTI ${ }^{2}$, ROSARIA D'URSO ${ }^{2}$, \\ PAOLO MERCANTINI ${ }^{1}$, GENOVEFFA BALDUCCI ${ }^{1}$ and MARCO CAVALLINI ${ }^{1}$ \\ ${ }^{1}$ Medical and Surgical Sciences and Translational Medicine, \\ Faculty of Medicine and Psychology "Sapienza", St. Andrea Hospital, Rome, Italy; \\ ${ }^{2}$ Department of Clinical and Molecular Medicine, Faculty of Medicine and Psychology, \\ University "Sapienza”, St. Andrea Hospital, Rome, Italy
}

\begin{abstract}
Background/Aim: Early gastric cancer (EGC) is usually associated with excellent prognosis. Some cases, however, entail a poorer survival. Our aim was to assess if EGC exfoliating into gastric lavage (GL) has a more aggressive behavior than the non-exfoliative counterpart. Patients and Methods: Between April 2012 and April 2017, 96 gastric cancer patients were prospectively submitted to preoperative GL to detect the presence (GL1) or absence (GLO) of exfoliated malignant cells. Results: A total of 16 patients had EGC. Tlb cases had significantly poorer overall $(O S)$, progression-free (PFS) and disease-free survival (DFS) than their GLO counterpart (16.3 vs. 61 months, $p=0.0032$ ). Similarly, the entire T1 class (T1a plus T1b EGCs) showed worse OS, PFS, DFS (15.5 vs. 61 months, $p=0.0008)$ and time-to-tumor progression (17 vs. 61 months, $p=0.0103$ ). Conclusion: In the case of EGC, the GLO-GL1 classification should become a routine clinical practice to identify the aggressive tumor phenotypes requiring for closer follow-up or additional treatment.
\end{abstract}

As of 2017, even though its registered incidence has been reduced over the last 20 years and the overall 5-year survival rate has improved over the last three decades, gastric cancer (GC) continues to be the third leading cause of cancer-

Correspondence to: Prof. Edoardo Virgilio, Medical and Surgical Sciences and Translational Medicine, Faculty of Medicine and Psychology "Sapienza", St. Andrea Hospital, via di Grottarossa 103539, 00189, Rome, Italy. Tel: +39 0633775693, Fax: +39 0633775322, e-mail: aresedo1992@yahoo.it; edoardo.virgilio@uniroma1.it

Key Words: Early gastric cancer, EGC, gastric lavage, gastric cancer oncology, gastric cancer cytopathology. related death in the world (1). Since the stage represents the most important prognostic factor for GC (at 5-year followup, $71 \%$ of treated GC patients with stage IA will be alive versus $4 \%$ of patients with stage IV), an early as well as rapid diagnosis is essential for extending life expectancy (2). Early gastric cancer (EGC), the initial condition in which the tumor is confined to the mucosa or submucosa (respectively, TIa and TIb cancer) undoubtedly represents the most desirable phase to detect the disease because of its usually excellent prognosis (5-year survival rates of over 90\%) (3). On the other hand, contrary to general expectations, a minority of EGC patients show a more aggressive phenotype $(4,5)$. The presence of loco-regional lymph node metastasis (LNM) represents the most important independent prognostic factor for EGC; more recently, other features such as lymphovascular invasion, tumor budding and mixed-type Lauren classification have been investigated and associated with higher risk of LNM and worse overall survival rates (69). Considering the paucity of information and available statistical data on such a topic, in this study we wanted to evaluate the correlation of EGC with a further parameter potentially indicative of aggressive neoplastic behavior: the presence versus absence of malignant cells exfoliated in the gastric lavage (GL) of EGC patients.

\section{Patients and Methods}

Study design. This study was conducted in accordance with the ethical standards of the 1964 Declaration of Helsinki and its later amendments. Between April 2012 and April 2017, 96 GC patients amenable to surgical resection were prospectively submitted to preoperative GL; of these, 38 subjects have already been included in our former studies on GC (10-12). All participants were from the Division of General and Emergency Surgery of St. Andrea Hospital, Faculty of Medicine and Psychology, University of Sapienza, Rome, 
Italy, and gave written consent before enrollment. The retrieved samples were shortly transported to the Cytological Department of our Hospital to be fixed, stained using the Papanicolau method and examined with a microscope. The presence or absence of cancer cells in GL (respectively, GLI and GL0) was then correlated with the classification and staging parameters traditionally recognized for GC and analyzed in terms of survival and prognosis; particular interest was directed to EGC cases. Pathologic verification of surgical specimens was described according to the 7th AJCC TNM Staging System (2).

Statistical analysis. Statistical analysis was performed using MedCalc Statistical Software for Windows, version 17.6 (MedCalc Software bvba, Ostend, Belgium). Continuous variables were analyzed through the Student's $t$-test whereas discrete data were compared using the Pearson's Chi-square test. Four types of survivals were entertained: median overall survival rate (OS) meaning the time elapsed from the day of GL to death from any cause, progression-free survival (PFS) going from sampling until metastatic (not recurrent) progression or death from any cause, time to tumor progression (TTP) differing from PFS for considering only cancer-related deaths and DFS or RFS (disease-free or recurrencefree survival) which is referred to the time between sampling and any recurrence or metastasis or death from any cause. All the survival curves were plotted using the Kaplan-Meier method and compared with the log rank test. Univariate analysis was performed using two-way ANOVA test while Cox proportional hazards model was used in multivariate analysis to assess independent prognostic factors. $p \leq 0.05$ was considered statistically significant.

\section{Results}

Clinicopathologic features of overall GC cases (including early and advanced tumors). 54 patients were men (56.2\%) and 42 women $(43.7 \%)$. Mean age was 66 years (range $=39$ 90). Forty-eight cancers were distal (antro-pyloric) and 48 proximal (cardial, corpo-fundic and gastric stump). Altogether, 93 patients (97\%) underwent surgery; after initial evaluation, multidisciplinary team scheduled palliative enteral feeding for two patients with advanced GC and endoscopic submucosal dissection (ESD) for one EGC patient. Among the surgical candidates, open surgery with curative intent was accomplished in 79 patients ( 35 total gastrectomies, 40 distal gastrectomies with Roux-en-Y reconstruction and 4 Billroth 2 partial gastrectomies); at surgery, 14 more patients were found with inoperable GC and were managed by palliative gastroenterostomy (2 cases), nutritional Witzel jejunostomy ( 8 cases) and palliative chemotherapy (4 cases). At histology, surgical margins of resected specimens resulted infiltrated in 9 cases (11.4\%). As for cancer grading, 12 patients had welldifferentiated (G1), 13 moderately differentiated (G2), 57 poorly differentiated (G3) and 14 undifferentiated carcinomas (G4). According to the Lauren classification, there were 54 intestinal (56.25\%), 28 diffuse (29\%) and 3 mixed (3\%) types of GC; classification was not declared for 11 cases (11.45\%). According to the 2010 WHO classification, 21 cancers were tubular, 8 mucinous, 6 poorly cohesive, 3 papillary, 3 with solid sheets; 25 cases (26\%) had signet-ring cells whereas data were not given for the remaining 30 cases. Lymphovascular (LVI) and peri-neural invasion (PnI) were present respectively in $57(59 \%)$ and 32 (33\%) instances. Tumor depth was as follows: 29 cases with T1-T2 (30.2\%) and 67 cases with T3-T4 (69.8\%). 70 patients had lymph node metastasis (N1, N2, N3a and N3b) (75\%) and 23 showed clinical and/or pathological distant metastasis (M1) (24\%). Metastatic lymph node ratio (LNR) was 0 in 26 cases (27\%), LNR1 (that is $>0-0.3$ ) in 28 patients (29\%), LNR2 $(>0.3-0.6)$ in $13(13.5 \%)$ and LNR3 (>0.6) in 29 cases (30\%). The pathologic stages were as follows: stage IA in 16 patients $(16.5 \%)$, IB in $6(6.25 \%)$, IIA in $3(3 \%)$, IIB in 9 (9\%), IIIA in $10(10.5 \%)$, IIIB in $20(21 \%)$, IIIC in $8(8 \%)$ and IV in 24 patients $(25 \%)$. Twenty-one patients received neoadjuvant treatment: of these, chemotherapy alone was administered in 17 (EOX, epirubicin-oxaliplatin-capecitabine; ECF, epirubicin-cisplatin-5 fluorouracil; and DCF, docetaxelcisplatin-5 fluorouracil) were the combinations more frequently adopted) and combined radio-chemotherapy in 4 cases. Forty-five patients were given adjuvant treatment: radio-chemotherapy was employed in 26 , chemotherapy alone in 17 and irradiation alone in 3 cases.

GLO/GL1 cytologic classification of GC cases. GL1 was present in 46 patients $(47.9 \%)$, GL0 in the remaining 50 subjects $(52.1 \%)$. Eight patients had GL1 after neoadjuvant treatment. GL1 correlated with statistical significanceto the parameters of tumor depth (T3-T4 vs. T1-T2), lymph node metastasis $(\mathrm{N}+v s$. N0), distant metastasis (M1 vs. M0), lymphovascular invasion (LVI1 vs. LVI0), peri-neural invasion (PnI1 vs. PnI0), Lauren classification (diffuse vs. intestinal histology), presence of signet-ring cells and administration of palliative treatment (respectively $p=0.0274, p=0.0324, p=0.0446, p=0.0287, p<0.0001$, $p=0.0413, p<0.0001, p=0.0184)$. Compared to the GL0 group, GL1 patients showed a statistically significant relative risk of developing advanced GC (T3-T4), lymph node and distant metastases, angiolymphatic and peri-neural invasion and signet-ring cells tumors (respectively $p=0.041$, $p=0.0481, p=0.0493, p=0.0301, p<0.0001$ and $p<0.0001)$.

GC patient's survival. After a median follow-up of 33.8 months (range $=2-62$ months) 55 patients have deceased: $53 \%$ of these (29 subjects) were GL1. Concerning the neoadjuvant subgroup, no statistical difference existed between GL1 and GL0 patients in terms of survival. Of the 41 patients alive in June 2017, one GL1 man developed recurrence at the anastomotic site and one more GL1 patient a metastatic liver disease. The median OS and PFS was of 18 and 32 months for GC patients respectively with positive and negative GL cytology $(p=0.017)$. Of interest, GL1 GC patients, compared to GL0 ones, had also shorter DFS (17.6 


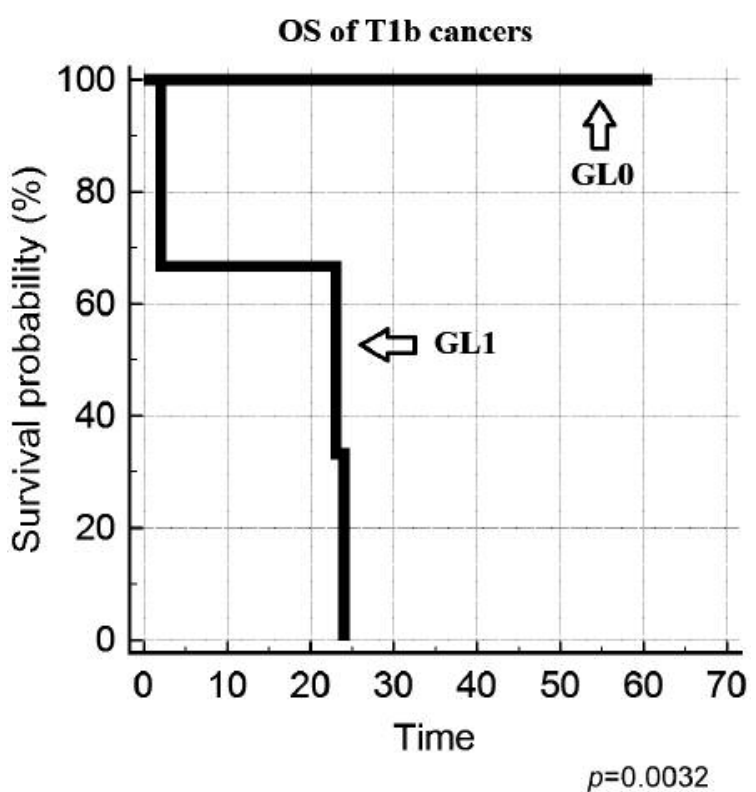

Figure 1. Overall survival (OS) of GL1 vs. GLO patients with T1b cancer. Considering the entire T1 class (T1a plus T1b), GL1 vs. GLO EGC had a significantly poorer OS, PFS and DFS (around $15.5 \mathrm{vs.} 61$ months, $p=0.0008$ ).

vs. 31.5 months, $p=0.0134)$ and TTP (19vs. 35 months, $p=0.056)$. The univariate model revealed a strong correlation between GL and OS and PFS $(p=0.021)$, DFS $(p=0.020)$ and TTP $(p=0.024)$. At multivariate analysis, GL1, Stage III-IV, M1, LVI1, PnI1, G3-G4 and necessity of adjuvant treatment resulted to be independent prognostic factor for poor OS (respectively $p=0.0287, p=0.0277, p=0.0161, p=0.0471$, $p=0.0299, p=0.0226$ and $p=0.0048)$. LVI1 was also the only multivariate independent factor for DFS $(p=0.0144)$ whereas no variable reached significance for PFS and TTP.

$E G C$. Of 96 GC patients, 16 had EGC (T1 cancer) and 80 an advanced tumor (AGC). As for tumor depth, of T1 cases, 7 were $\mathrm{T} 1 \mathrm{a}(7.3 \%)$ and $9 \mathrm{~T} 1 \mathrm{~b}(9.3 \%)$. All lesions were treated with gastric resection (9 distal and 6 total gastrectomies) except for one T1b case treated with ESD. Of the 55 GC deaths occurred as of June 2017, 4 patients had EGC: of note, all of these were GL1. One patient had a post-neoadjuvant GL1 T1a tumor and 3 patients were GL1 T1b cancer (1 GL was collected after neoadjuvant treatment). The deceased T1a patient was a 75-year-old cirrhotic woman with a cardial GL1 EGC previously treated with neaodjuvant chemo-radiotherapy and total gastrectomy who developed anastomotic recurrence and peritoneal carcinomatosis at 7 month-follow-up. As for T1b dead cases, 1 woman with lymph node metastasis (the only case T1 N1) deceased 23 months after distal gastrectomy followed by adjuvant chemo-radiotherapy and subsequent bilateral salpingo-oophorectomy for Krukenberg tumor; 1 man deceased 24 months after distal gastrectomy for cerebrovascular accident following an urologic intervention and one more male deceased 1 month following complete gastrectomy due to respiratory failure and sepsis. Considering only EGC cases, there was no statistical association between $\mathrm{T}$ status and the other clinicopathologic features except for a positive trend with differentiation $(p=0.0231)$. At Kaplan-Meier curves of T1a subgroup, there was no statistical difference between GL0 and GL1 patients in terms of OS, PFS, DFS and TTP. On the other hand, compared to their GLO counterpart, EGC patients with GL1 T1b met with significantly shorter OS, PFS, DFS (approximately 16.3 vs. 61 months, $p=0.0032$ ) (Figure 1). Furthermore, considering the entire T1 class (T1a plus T1b), GL1 vs. GL0 EGC had a significantly poorer OS, PFS and DFS (around 15.5 vs. 61 months, $p=0.0008$ ) (Figure 2a) as well as an earlier TTP (respectively 17 vs. 61 months, $p=0.0103$ ) (Figure 2b).

\section{Discussion}

As of 2017, some features of EGC have been well established; other data, however, need further clarification. Among the former, the usually excellent prognosis (5-year survival rates of over 90\%), discrepant incidence between Asian (higher rates) and Western countries (where screening programs are absent), the importance of detecting associated lymph node metastases and the large available choice of treatment modalities (traditional versus laparoscopic surgery with or without sentinel lymph node biopsy, endoscopic mucosal versus submucosal dissection) have been well described in the pertinent literature through the years $(3,13$, 14). On the other hand, the meaning of EGC as well as its prognostic characteristics have not been well defined yet. EGC definition provided by the Japanese classification, referring to a tumor limited to mucosa (T1a) or submucosa (T1b) irrespective of the presence of lymph node metastasis, seems to us the most appropriate for this condition; in the past, in fact, EGC has been generally referred to "nonadvanced" tumors (including T2) and "non-advanced" stages of disease including T1N0M0 (stage IA), T1N1M0 and T1N0M0 (stage IB) $(14,15)$. Furthermore, the classification of GC provided by the 7th edition of the AJCC staging system appears to be inadequate for EGC since it was based on advanced GC and did not focus on a staging system for $\mathrm{T} 1$ cancer (16). In less than $10 \%$ of cases, EGC entails a worse survival in comparison with the one generally expected: the coexistence of LNM, which has been assessed as an independent risk factor for EGC recurrence and the most valuable prognostic factor for EGC, is a certain cause of this phenomenon but it could not act alone $(4-6,16)$. More recently, in fact, other features such as lymphovascular invasion, tumor budding and mixed-type Lauren classification have been investigated and associated with higher risk of 
a

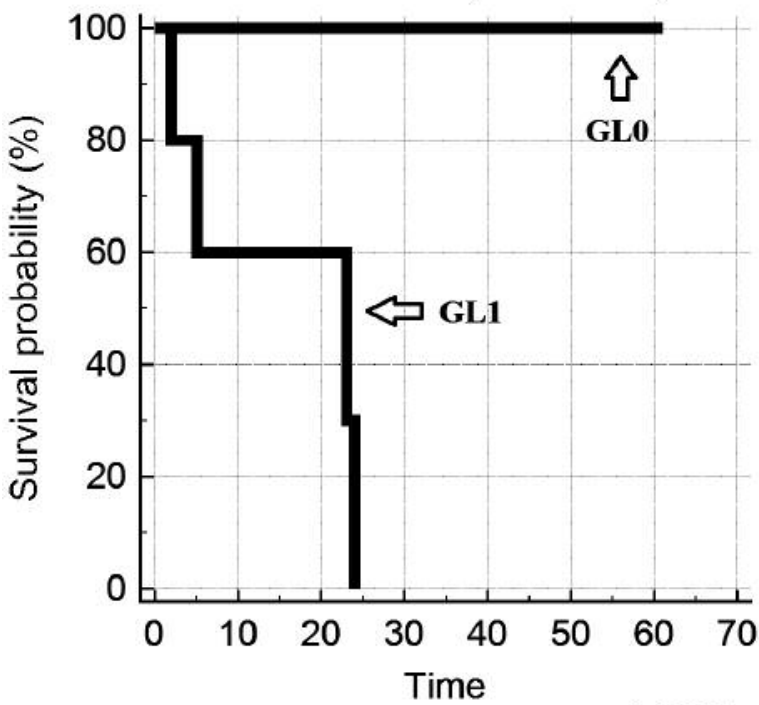

$p=0.0008$

b

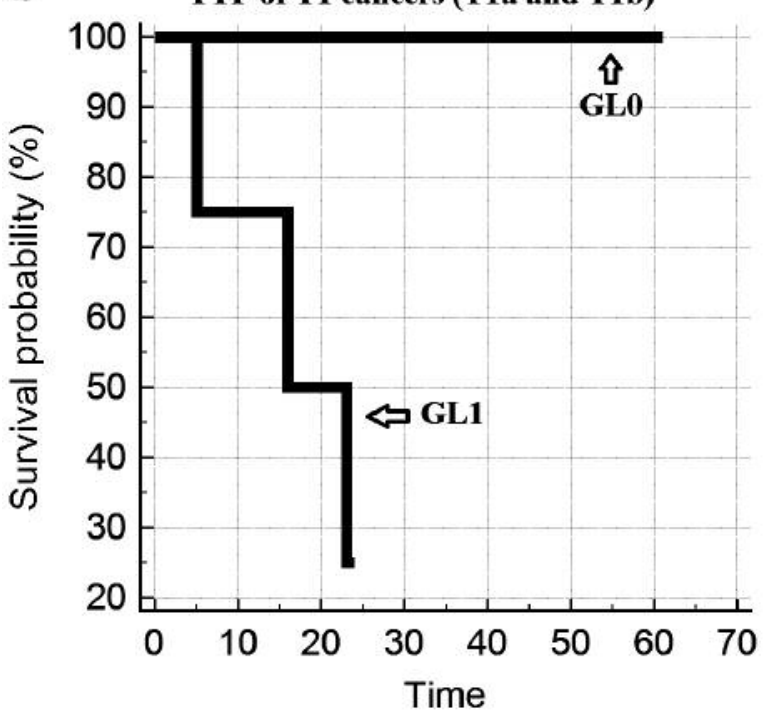

$p=0.0103$

Figure 2. a) OS of GL1 vs. GLO patients with T1 cancer (T1a plus T1b). b) TTP of GL1vs. GLO patients with EGC cancer (T1a plus T1b).

LNM and worse overall survival rates (6-9). Since malignant mucosal cells, following the sixth metastatic route (Figure 3), can be found in stomach juice and GL of GC patients and have been also associated with particularly aggressive phenotypes of cancers, for our study we elected GL as biological material to be cytologically analyzed with staging and prognostic intent $(17,18)$. Following analysis of $96 \mathrm{GC}$ patients (16 EGCs plus 80 AGCs), we assessed GL1 as indicative of aggressive tumor phenotype: compared to GL0

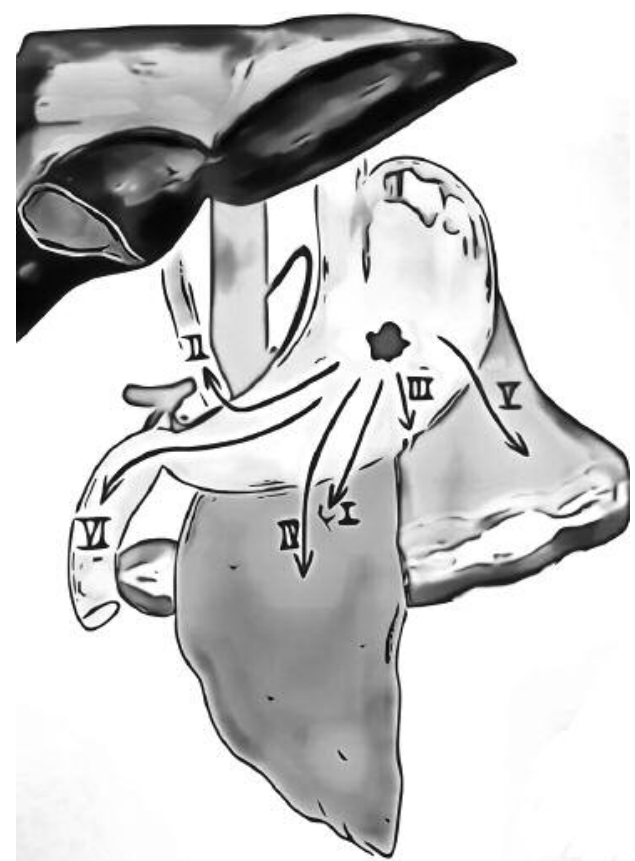

Figure 3. Illustration of the six metastatic routes followed by GC. I: direct infiltration of contiguous structures; II: hematogenous invasion; III: lymphatic metastasis; IV: intraperitoneal dissemination; V: mesogastrium spread; VI: endoluminal exfoliation.

counterpart, in fact, GL1 correlated with statistical significance to many other aggressive parameters (advanced tumor depth, lymph node and distant metastases, angiolymphatic and peri-neural invasion, Lauren diffuse type, presence of signet-ring cells and necessity of palliative treatment - respectively, $p=0.0274, p=0.0324, p=0.0446$, $p=0.0287, p<0.0001, p=0.0413, p<0.0001, p=0.0184)$, entailed worse OS, PFS, DFS, TTP (approximately $18 v s .32$ months, $p<0.05)$ and resulted to be an independent prognostic factor for poor OS at multivariate analysis $(p=0.0287)$. In addition, focusing on survival of T1 cancers, we found that GL1 T1b EGCs registered significant differences in OS, PFS, DFS (approximately $16.3 v s .61$ months, $p=0.0032$ ) (Figure 1). Moreover, concerning $\mathrm{T} 1$ overall class (that is T1a plus T1b), GL1 vs.GL0 EGC had a significantly poorer OS, PFS, DFS (around $15.5 v s .61$ months, $p=0.0008$ ) (Figure 2a) as well as a more precocious TTP (respectively 17 vs. 61 months, $p=0.0103$ ) (Figure 2b). In the light of our results we consider GL1 as a feature of aggressive phenotype not only for AGC but also for EGC and, with this motivation, encourage the clinical use of GL0-GL1 classification.

\section{Conflicts of Interest}

The Authors declare no conflict of interest. 


\section{References}

1 Torre LA, Bray F, Siegel RL, Ferlay J, Lortet-Tieulent J and Jemal A: Global cancer statistics, 2012. CA Cancer J Clin 65: 87-108, 2015.

2 Edge SB, Byrd DR, Compton CC, Fritz AG, Greene FL and Trotti A: AJCC Cancer Staging Handbook. From the AJCC Cancer Staging Manual. 7th ed. New York, Springer-Verlag, 2010.

3 Sano T, Katai H, Sasako M and Maruyama K: The management of early gastric cancer. Surg Oncol 9: 17-22, 2000.

4 Kobayashi M, Araki K, Matsuura K, Kawai S and Moriki T: Early gastric cancer giving rise to bone and brain metastases-a review of the Japanese literature. Hepatogastroenterology 49: 1751-1754, 2002.

5 Kakushima N, Kamoshida T, Hirai S, Hotta S, Hirayama T, Yamada J, Ueda K, Sato M, Okumura M, Shimokama T and Oka Y: Early gastric cancer with Krukenberg tumor and review of cases of intramucosal gastric cancers with Krukenberg tumor. J Gastroenterol 38: 1176-1180, 2003.

6 Barreto SG and Windsor JA:Redefining early gastric cancer. Surg Endosc 30: 24-37, 2016.

7 Gulluoglu M, Yegen G, Ozluk Y, Dogan S, Gundogdu G, Onder S, Balik E: Tumor budding is independently predictive for lymph node involvement in early gastric cancer. Int J Surg Pathol 23: 349-359, 2015.

8 Pyo JH, Lee H, Min BH, Lee JH, Choi MG, Lee JH, Sohn TS, Bae JM, Kim KM, Yeon S, Jung SH, Kim JJ and Kim S: Early gastric cancer with a mixed-type Lauren classification is more aggressive and exhibits greater lymph node metastasis. J Gastroenterol 52: 594-601, 2017.

9 Huh CW, Jung DH, Kim JH, Lee YC, Kim H, Kim H, Yoon SO, Youn YH, Park H, Lee SI, Chiu SH, Cheong JH and Noh SH: Signet ring cell mixed histology may show more aggressive behavior than other histologies in early gastric cancer. J Surg Oncol 107: 124-129, 2013.

10 Virgilio E, Proietti A, D'Urso R, Cardelli P, Giarnieri E, Montagnini M, Giovagnoli MR, Mercantini P, Balducci G and Cavallini M: Measuring intragstric tumor markers in gastric cancer patients: a systematic literature review on significance and reliability. Anticancer Res 37: 2817-2821, 2017.

11 Virgilio E, Giarnieri E, Montagnini M, D’Urso R, Proietti A, Mesiti A, Giovagnoli MR, Mercantini P, Cavallini M and Balducci G: Detection of cancer cells and tumor markers in gastric lavage of patients with gastric cancer: do these findings have a clinicopathological significance and oncological implication? Med Hypotheses 94: 1-3, 2016.
12 Virgilio E, Giarnieri E, Montagnini M, D’Urso R, Proietti A, Mesiti A, Giovagnoli MR, Mercantini P, Cavallini M and Balducci G: Analyzing gastric lavage of gastric cancer patients: a prospective observational study on cytopathology and determination of intragastric CEA, Ca 19.9, Ca 72.4 and Ca 50. Acta Cytol 60: 161-166, 2016.

13 Probst A, Schneider A, Schaller T, Anthuber M, Ebigbo A and Messmann H: Endoscopic submucosal dissection for early gastric cancer: are expanded resection criteria safe for western patients? Endoscopy, 2017. doi: 10.1055/s-0043-110672. [Epub ahead of print]

14 Bollschweiler E, Berlth F, Mönig S and Hölscher AH: Treatment of early gastric cancer in the Western World. World J Gastroenterol 20: 5672-5678, 2014.

15 Japanese Gastric Cancer Association: Japanese classification of gastric carcinoma - 2nd English Edition. Gastric Cancer 1: 1024, 1998.

16 Choi KH, Kim BS, Oh ST, Yook JH and Kim BS: Comparison the sixth and seventh editions of the AJCC staging system for T1 gastric cancer: a long-term follow-up study of 2124 patients. Gastric Cancer 20: 43-48, 2017.

17 Watanabe Y, Kim SH, Castoro RJ, Chung W, Estecio MR, Kondo K, Ahmed SS, Toyota M, Itoh F, Suk KT, Cho MY, Shen L, Jelinek J and Issa JP: Sensitive and specific detection of early gastric cancer using DNA methyltion analysis of gastric washes. Gastroenterology 136: 2149-2158, 2009.

18 Nakajima T, Konishi H, Tatsumi Y, Sakamoto Y, Yamane Y, Misawa S, Toyama S, Ochiai T, Kashima K, Konishi E and Tsuchihashi Y: Gastric cancer presenting with extremely rapid growth: unprecedented morphologic change in a short time and endoscopic estimation of its doubling time. Endoscopy 32: 994$997,2000$.
Received April 5, 2017

Revised May 5, 2017

Accepted June 5, 2017 